\title{
Leishmaniasis of the ear treated successfully by photodynamic therapy
}

\author{
Hanane Bay Bay*, Sara Elloudi, Selma Benkirane, Zakia Douhi, Salim Gallouj and Fatima Zahra Mernissi \\ University Hospital Center, Fez, Morocco
}

\begin{abstract}
Introduction: Cutaneous leishmaniasis is a parasitic infection characterized by unusual clinical aspects, sometimes on in the immunodeficient patient or associated with particular parasitic species. We report an unsual case treated by the Photodynamic therapy (PDT) successfully.

Case report: Patient aged 43, followed for haemorrhagic rectocolitis (HCR) for 4 years treated by Infliximab and Azathioprine, present since one and a half years of a pruriginous and slightly painful crustal lesion of centrifugal evolution in the right ear. The first cutaneous biopsy was in favor of pyoderma gangrenosum. The patient was on systemic corticosteroids. The evolution was marked by the installation of a perichondritis and the appearance of new lesions in the face and neck. Dermoscopic examination suggested cutaneous leishmaniasis wich was confirmed histologically. The patient was on intramuscular pentavalent antimonial drugs for one month and Chlortetracyclin $3 \%$ ointment and with partial response. Because of the push of HCR, the treatment by PDT was indicated with good evolution. Indeed, there was complete regression of the lesions with a satisfactory aesthetic result.
\end{abstract}

Discussion/Conclusion: Anti tumour necrosis factor $\alpha$ can promote unusual and the occurrence of leishmaniasis which illustrates our case. PDT is a good therapeutic alternative resistant form of classical drugs.

\section{Introduction}

Cutaneous Leishmaniasis (CL) are zoonoses caused by parasites of the genus Leishmania, transmission occurs by bite of a gnat called sandfly. It is a worldwide health problem. In fact, The World Health Organization (WHO) considers leishmaniasis to be one of the most serious parasitic diseases including Morocco because of the global warming [1,2]. In Morocco, major leshmaniasis, tropica and infantum are prevalent and are very common [2]. Among the problems reported in the treatment of cutaneous leishmaniosis are difficulty in determining clinical diagnosis due to lack of access tomicroscopy onmany basic health services [3]; the serious side effects of pentavalent antimonial drugs and the variability of the efficacy against the different forms of Leishmania [3], making drugs and medical attention an expensive treatment because of the side effects [3]; besides, there have also been reports of patients not responding to drugs due to drug resistance or increased immunosuppression [3]. Photodynamic Therapy (PDT) is non-invasive therapy and can be repeated when necessary without damage to the patient's health and without causing resistance in the parasite [4-7]. We report a case illustrate this difficulty to diagnosis and treat leshmaniasis in the immunocompromised who was treated successfully by (PDT).

\section{Case Report}

This was a 43-year-old patient from the north of Morocco who has been followed for ulcerative colitis for 4 years under INFLIXIMAB and AZATHIOPRINE. For the last year and a half, he has had a pruriginous and slightly painful crusted lesion of centrifugal evolution of the right ear. He had benefited from a cutaneous biopsy in favor of Pyoderma gangrenosum. The patient was on oral and injectable corticosteroids, antibiotics and local care with no improvement. The evolution was marked by the installation of a perch chondritis and the appearance of new ulcerated plaques and nodules in the face and neck (Figures 1-4). Dermoscopic examination revealed tears, a white star-like appearance, salmon red areas, polymorphic vascularization, scales and scabs (Figures 5 and 6). All lesions suggestive leshmaniasis and second biopsie was indicated. The histology was confirmed leishmaniasis (Figures 7 and 8). In our case, we have had multifocal lesions, an iatrogenic immunosuppression without visceral involvement. So, our decision was stopping immunosuppressants and treating by pentavalent antimonial $35 \mathrm{mg} / \mathrm{kg} / \mathrm{d} / 20$ days with Chlortetracyclin ointment $3 \%$ for 1 month, then, Gentamycin ointment $0.5 \%$, blue light and cryotherapy except the ear. A moderate elevation of lipasemia and amylasemia was marked. The evolution was marked by a partial improvement of the lesion of the ear with moderate regression of edema and erythema and disappearance of lesions of the nose and neck with persistent lesions of the cheek, and the neck (Figures 9-12). At the dermoscopic control, we always noted the structures in tears in favor of persistence of leishmani bodies. Skin smear in search of leshmaniasis cells was positive. However, our patient had a flare-up of his ulcerative colitis disease and we have to quickly control the infection in order to resume immunosuppressive therapy. So we opted for 2 sessions of PDT at one week intervals. The photosensitizing agent was Methyl aminolevulinate and after 3 hours, the lesions were exposing to a red light source, we use Waldman PDT $1200 \mathrm{~L}$ as $100 \mathrm{j} / \mathrm{cm}^{2}$. The pain was tolerated because of Verbal analgesia, premedication by paracetamol and using of the cold. The suites were simple. PDT allowed complete regression of lesions

*Correspondence to: Hanane Bay Bay, University Hospital Center, Fez, Morocco, E-mail: hananebaybay@gmail.com

Received: April 18, 2018; Accepted: April 25, 2018; Published: April 30, 2018 


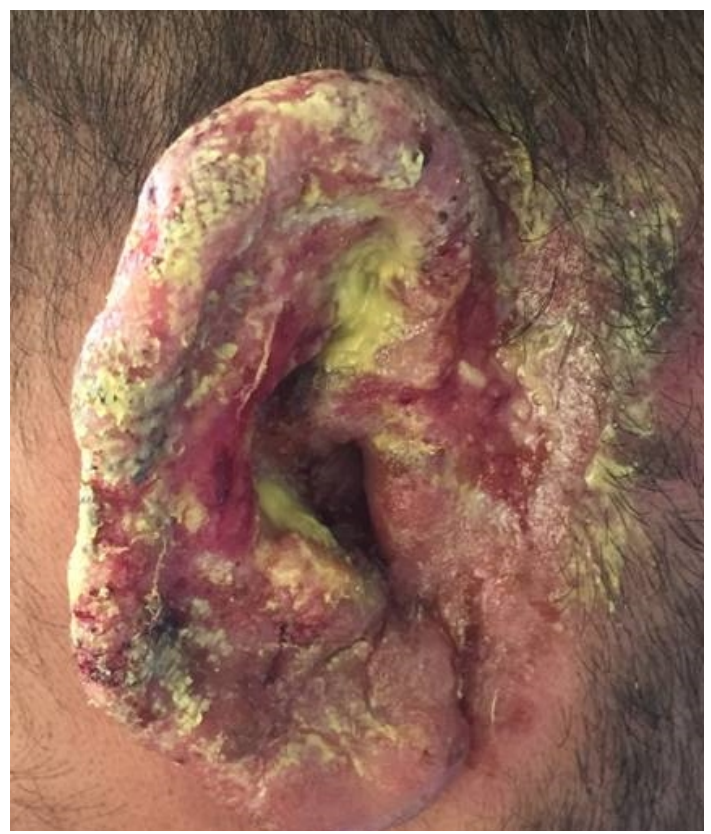

Figure 1. Ulcerative and crusted lesion in the right ear

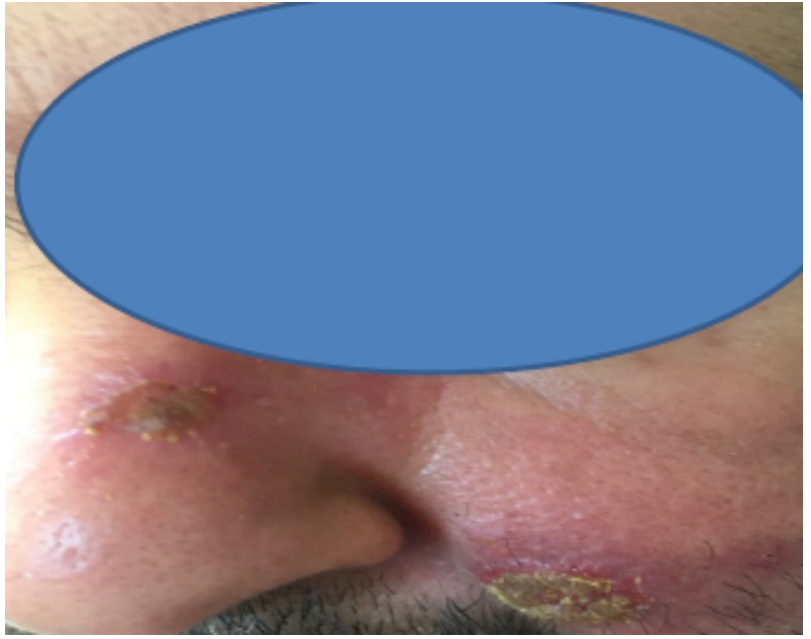

Figure 2. Crusted and ulcerated nodular in the nose and check

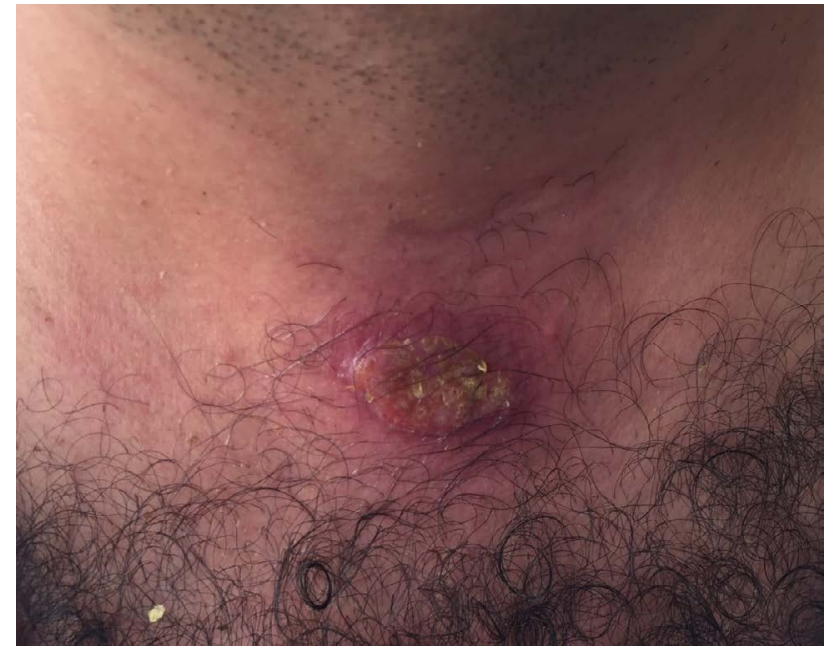

Figure 3. Crusted and ulcerated plaque in the trunk 


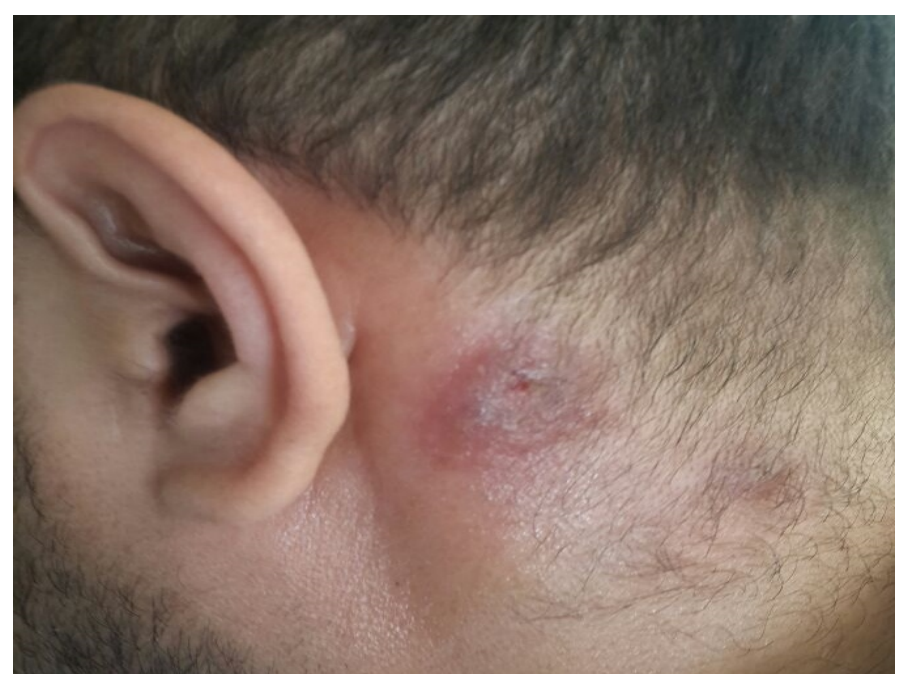

Figure 4. Crusted and ulcerated plaque in the neck

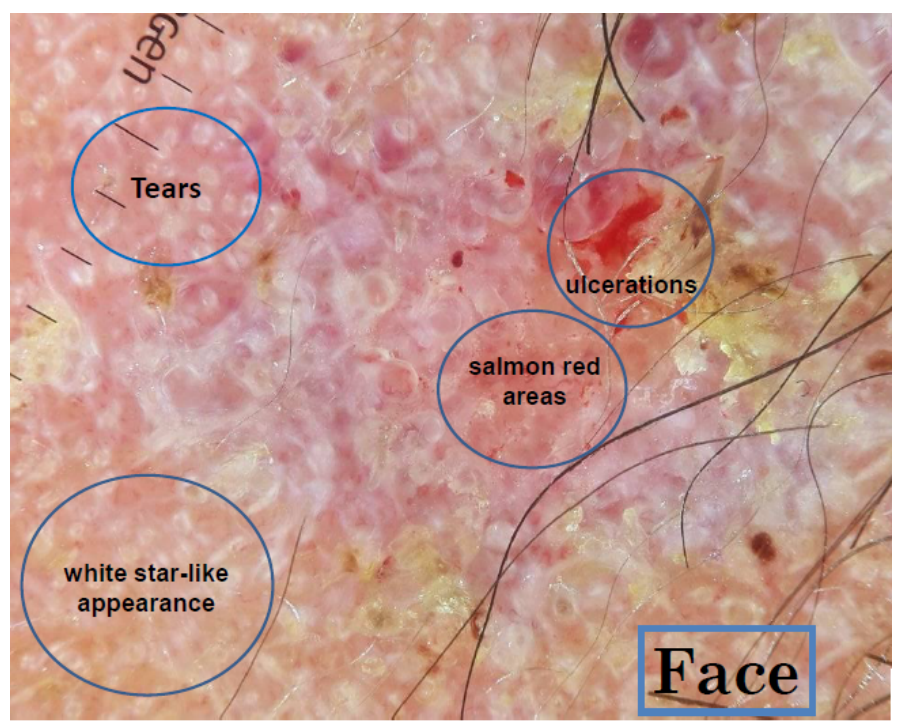

Figure 5. Dermoscopy appearance of the face lesion

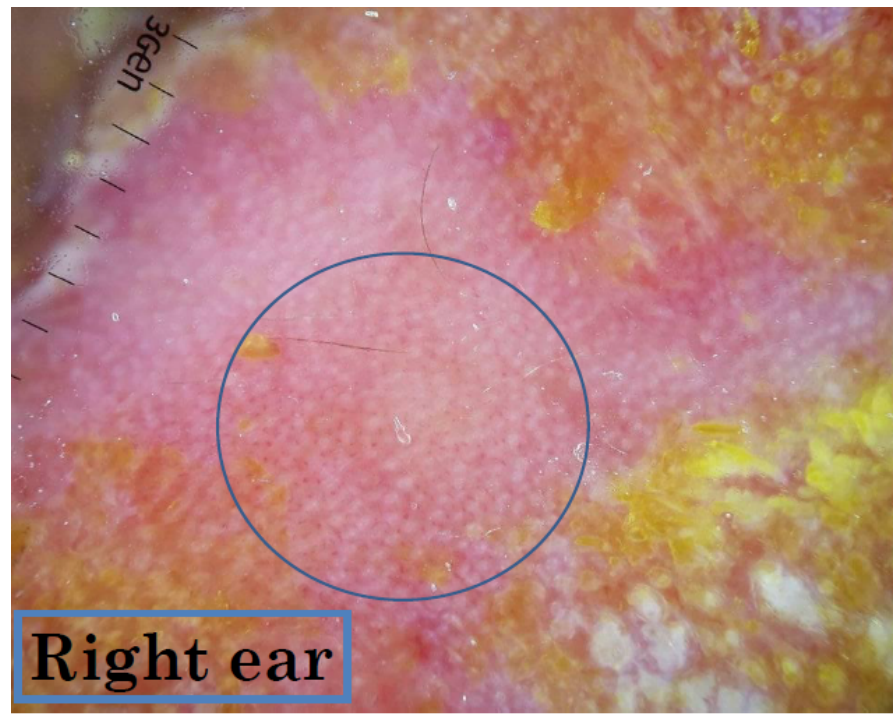

Figure 6. Dermoscopy appearance of the ear lesion

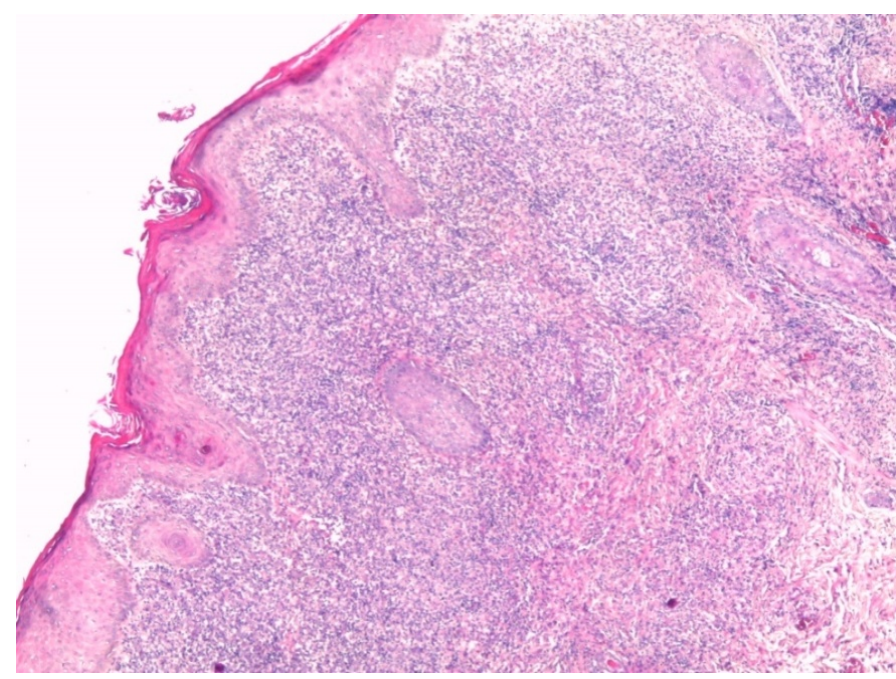

Figure 7. Histology of the lesion with granuloma

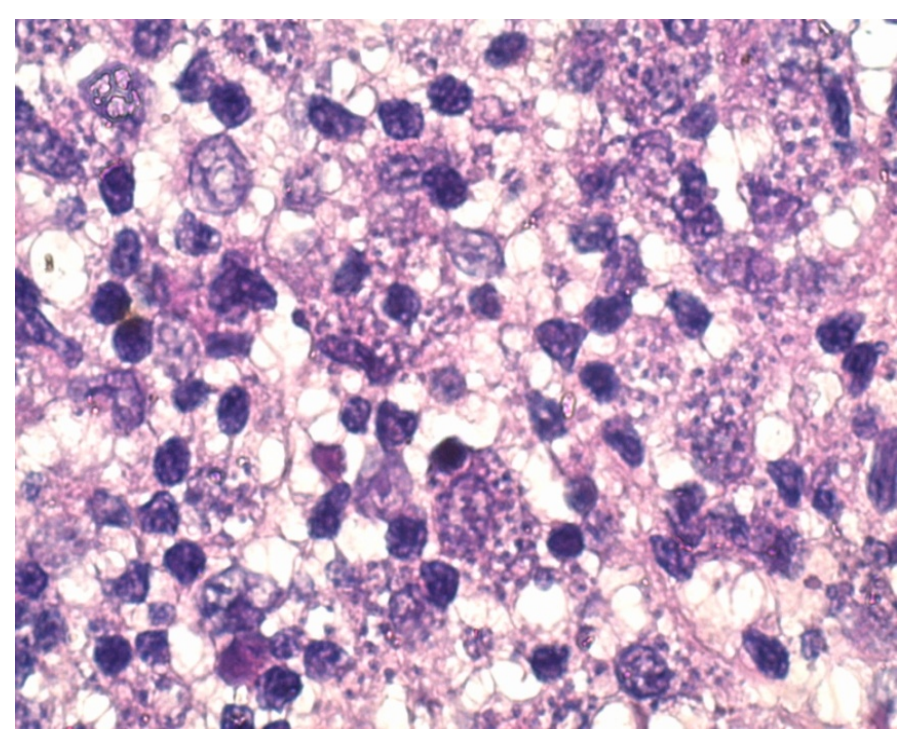

Figure 8. Histology of the lesion with leshmania body

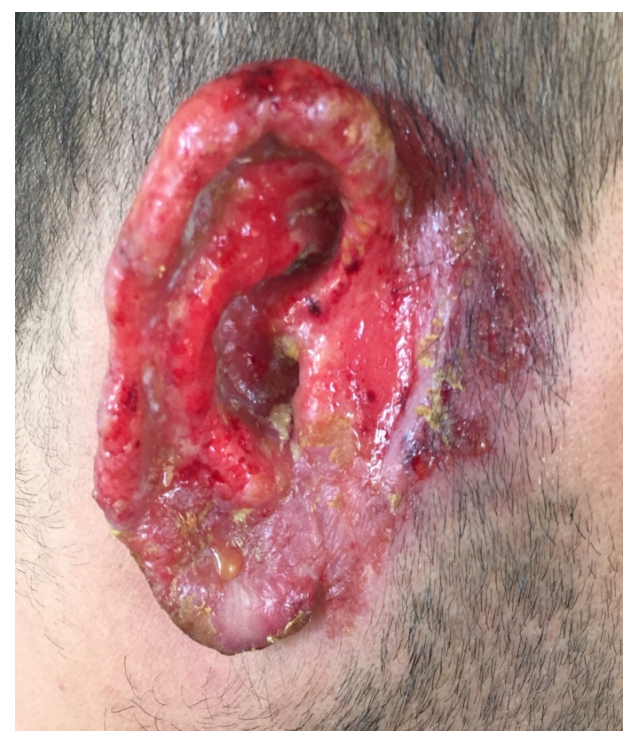

Figure 9. Partial response in the ear 


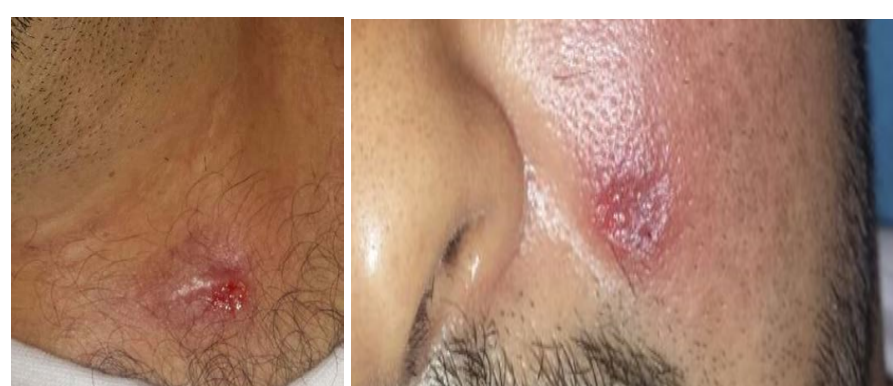

Figure 10. Partial response in the trunk and check

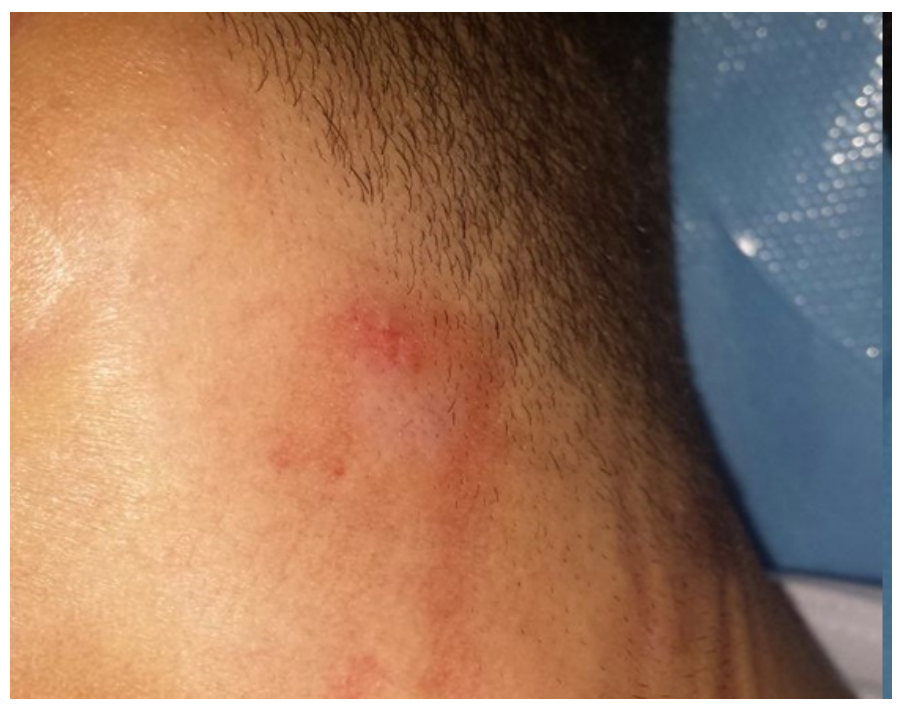

Figure 11. Partial response in the neck

with a good aesthetic resultas you can see in picture without reccurence after 1 year (Figures 13-15).

\section{Discussion}

Cutaneous leishmaniasis (CL) is a parasitic infection characterized by significant clinical polymorphism. The diagnosis must be evoked in case of persistent skin lesions, ulcerated or nodular, returning from an endemic area $[1,3]$. Unusual clinical features have been described, sometimes occurring on immunodeficient sites or associated with particular parasitic species [1,3]. In our case, the isolated localization at the beginning of the ear was confusing. The immunosuppressive treatment contributed to the extension of the disease, but, persistence of symptomatology, appearance of new lesions in the discovered area, dermoscopic appearance and histology have rectified the diagnosis of cutaneous leshmaniasis. Experimental studies have shown that anti tumour necrosis factor $\alpha$ (Anti -TNF $\alpha$ ) can promote the occurrence of leishmaniasis [8] which illustrates the case of our patient. (Anti TNF $\alpha$ ) caused deficiency of an inflammatory response and induced atypical clinical forms. The Sodium stibogluconate is the gold standard of leshmaniasis treatment. However, this drug is quite toxic to humans, expensive, with emergence of resistance cases for this treatment. Others alternative of treatment in Morocco specially in the persistent or resistant forms are Amphetericine B whitch not diponible in our country and Fluconazole, Metronidazole, Clarythromycin, Doximycin with Variable result [3,9]. Clinical studies have shown that, in contrast with systemic treatment, PDT has no or low signs of toxicity and excellent esthetic results have been obtained in lesions of patients with CL [4-7], thus solving the tissue damage associated with previous treatment options without drug resistance

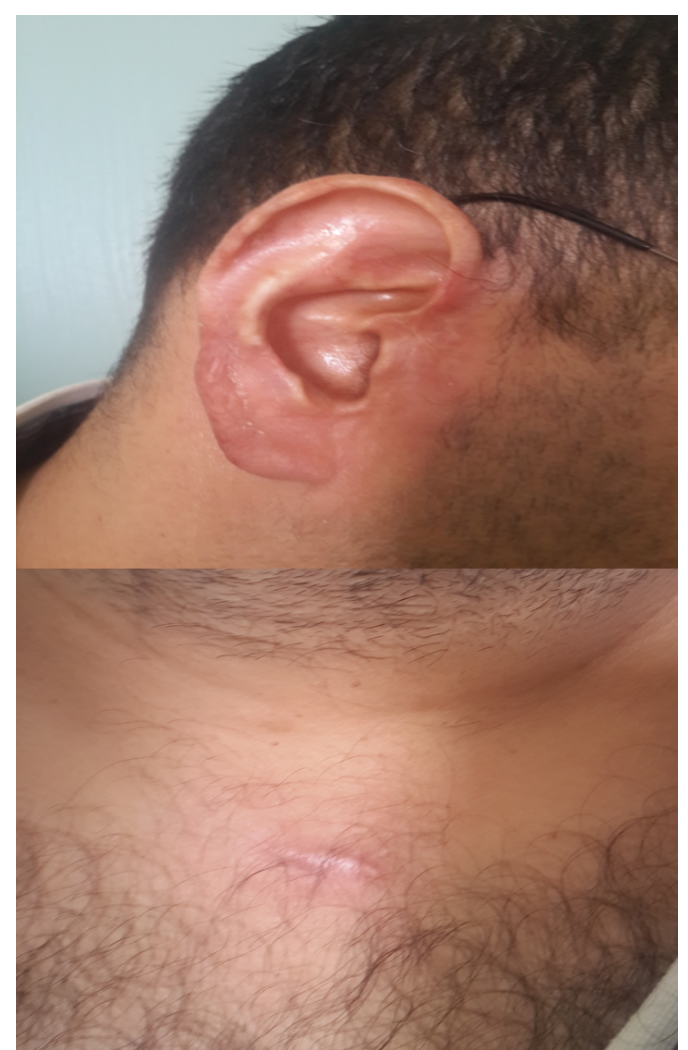

Figures 12, 13. Complete response in the right ear and trunk

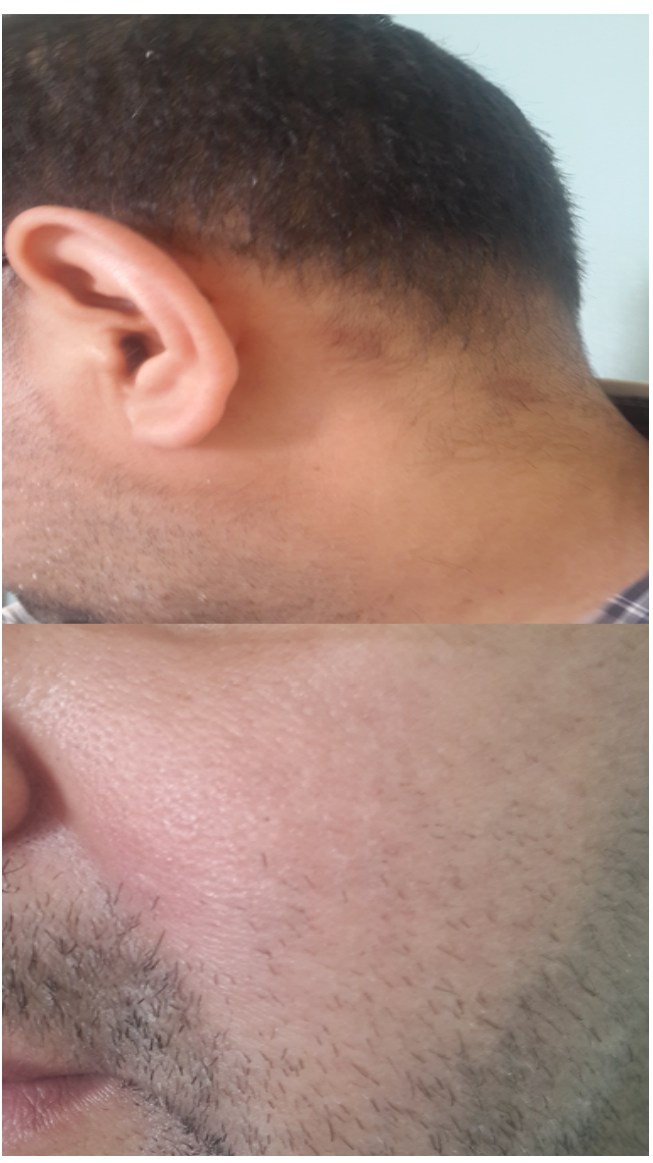

Figures 14, 15. Complete response in the neck and check 
PDT mechanism of action are based on a photosensitive substance that are stimulated to energize stage, when back to the ground stage its energy is transferred to oxygen to produce oxygen free radical or singlet oxygen responsible for cell necrosis [4-7], a photosensitive substance was intralesional aminolevulinic acid in our case. Also, recently, the methylene blue could be used as photosensitizer with good result [6]. For our patient, despite its high cost, the PDT allows the fastest control, whatever the responsible species. Two sessions only were enough to have a quick control without signs of toxicity, good aesthetic results. We opted for this indication because of incomplete response and the necessity of resumption of immunosuppressive therapy for the new push of ulcerative colitis.

\section{Conclusion}

Immunocompromised patients have atypical forms of cutaneous leishmaniasis. It must be evoked about any chronic ulceration on a discovered area, resistant to treatment. Dermoscopyguids the diagnosis and allows monitoring during treatment. Finally, we think PDT is a good therapeutic alternative for multi-lesional leishmaniasis and periorificial localization.

\section{References}

1. Oumeish OY, Parish LC (1999) Cutaneous leishmaniasis (SLCL) is an important public health problem in many countries. Clin Dermatol 17: 247-8. [Crossref]

2. El Alami S, Aoufi S (2015) [88 years of cutaneous leishmaniasis in Morocco]. Med Sante Trop 25: 97-101. [Crossref]

3. Minodier P, Parola P (2007) Cutaneous leishmaniasis treatment. Travel Med Infect Dis 5: 150-158. [Crossref]

4. Enk CD, Nasereddin A, Alper R, Dan-Goor M, Jaffe CL, et al. (2015) Cutaneous leishmaniasis responds to daylight-activated photodynamic therapy: proof of concept for a novel self-administered therapeutic modality. Br J Dermatol 172: 1364-70. [Crossref]

5. Souza DM, Alves PM, Silva ML, Paulino TP, Coraspe HO, et al. (2017) 5-ALAmediated photodynamic therapy reduces the parasite load in mice infected with Leishmania braziliensis. Parasite Immunol 39. [Crossref]

6. De Oliveira S, da Ordem Trahamane EJ, Monteiro J, Santos GP, Crugeira P, et al (2017) Leishmanicidal effect of antiparasitic photodynamic therapy-ApPDT on infected macrophages. Lasers Med Sci 32: 1959-1964. [Crossref]

7. Evangelou G, Krasagakis K, Giannikaki E, Kruger-Krasagakis S, Tosca A (2011) Successful treatment of cutaneous leishmaniasis with intralesional aminolevulinic acid photodynamic therapy. Photodermatol Photoimmunol Photomed 27: 254-6. [Crossref]

8. Marcoval J, Penín RM, Sabé N, Valentí-Medina F, Bonfill-Ortí M, et al. (2017) Cutaneous leishmaniasis associated with anti-tumour necrosis factor-a drugs: an emerging disease. Clin Exp Dermatol 42: 331-334. [Crossref]

9. Veraldi S, Nazzaro G2 (2016) Successful treatment of cutaneous leishmaniasis of the ear with oral fluconazole. Clin Exp Dermatol 41: 315-316. [Crossref]

Copyright: $(\mathcal{C} 2018$ Bay HB. This is an open-access article distributed under the terms of the Creative Commons Attribution License, which permits unrestricted use, distribution, and reproduction in any medium, provided the original author and source are credited. 